\title{
A importância do cirurgião dentista na unidade de terapia intensiva
}

\author{
The importance of dental surgery in the intensive care unit
}

La importancia de la cirugía dental en la unidad de cuidados intensivos

José Milton de Aquino e Silva Neto ${ }^{1 *}$, João Pedro Matar Lemos ${ }^{1}$, Celso Pereira do Nascimento', Caio César Abreu Celestino', Maria Eduarda Brandão Balbino Torres' ${ }^{1}$, Michelle Leão Bittencourt Brandão Medeiros ${ }^{1}$, Tayguara Cerqueira Cavalcanti'.

\section{RESUMO}

Objetivo: Destacar a relevância do Cirurgião Dentista (CD) na Unidade de Terapia Intensiva (UTI) focandose na execução dos cuidados da cavidade bucal desses pacientes. Revisão bibliográfica: Pacientes que encontram-se na UTI devido inúmeras intercorrências e não apresentam uma boa higienização da cavidade bucal, possuem uma maior potencialidade de possuir um resultado ruim na evolução do seu quadro clínico. Esse acontecimento na maioria das vezes é aumentado pela percentagem de problemas respiratórios. É constatado que a prevalência de insucesso no quadro evolutivo é maior nos pacientes que não possuem uma determinada higienização na cavidade bucal. Geralmente na maioria dos hospitais essa limpeza é feita por profissionais de enfermagem dos quais não apresentam compreensão de forma aprofundada ou até mesmo protocolos para nortear, o que estabelece um indicativo da importância do CD na constituição interdisciplinar, intervindo assim com um melhor senso crítico e embasamento cientifico. Considerações finais: Nos dias atuais o CD vem buscando se qualificar ainda mais nas intervenções dos processos reabilitadores em indivíduos que encontram-se na UTI, procurando diminuir focos infecciosos na cavidade bucal, realizando diversos procedimentos, melhorando dessa forma o quadro de saúde do paciente e diminuindo o tempo de internação dos mesmos na UTI.

Palavras-chave: Equipe hospitalar de odontologia, Assistência ao paciente, Infecção hospitalar.

\begin{abstract}
Objective: Highlight the relevance of the Dental Surgeon (CD) in the Intensive Care Unit (UTI) focusing on the care of the oral cavity of these patients. Literature review: Patients who are in the UTI due to numerous complications and do not have a good hygiene of the oral cavity, have a greater potential to have a bad result in the evolution of their clinical condition. This event is most often increased by the percentage of breathing problems. It is found that the prevalence of failure in the evolutionary picture is higher in patients who do not have a certain hygiene in the oral cavity. Generally in most hospitals nursing professionals who do not have a thorough understanding or even protocols to guide do this cleaning, which establishes an indication of the importance of the $C D$ in the interdisciplinary constitution, thus interfering with a better critical sense and foundation scientific. Final considerations: Nowadays the $C D$ is seeking to qualify even more in the interventions of the rehabilitation processes in individuals who are in the UTI, seeking to reduce infectious foci in the oral cavity, performing various procedures, thus improving the patient's health and reducing the time hospitalization in the UTI.
\end{abstract}

Keywords: Dental staff hospital, Patient care, Cross infection.

${ }^{1}$ Centro Universitário CESMAC (CESMAC), Maceió - AL.

*E-mail: milton_neto_166@hotmail.com

SUBMETIDO EM: 9/2020

ACEITO EM: 10/2020

PUBLICADO EM: 1/2021 


\section{RESUMEN}

Objetivo: Destacar la relevancia del Cirujano Dentista (CD) en la Unidad de Cuidados Intensivos (UTI) enfocándose en el cuidado de la cavidad bucal de estos pacientes. Revisión bibliográfica: Los pacientes que se encuentran en la UTI por numerosas complicaciones y no tienen una buena higiene de la cavidad bucal, tienen un mayor potencial de tener un mal resultado en la evolución de su cuadro clínico. Este evento suele aumentar con el porcentaje de problemas respiratorios. Se encuentra que la prevalencia de falla en el cuadro evolutivo es mayor en pacientes que no tienen una cierta higiene en la cavidad bucal. Generalmente en la mayoría de los hospitales esta limpieza es realizada por profesionales de enfermería que no tienen un conocimiento profundo ni tampoco protocolos para orientar, lo que establece una indicación de la importancia del CD en la constitución interdisciplinaria, interviniendo así con un mejor sentido crítico y fundamento. científico. Consideraciones finales: Hoy en día el CD busca capacitar aún más en las intervenciones de los procesos de rehabilitación en las personas que se encuentran en la UTI, buscando reducir los focos infecciosos en la cavidad bucal, realizando diversos procedimientos, mejorando así la salud del paciente y reduciendo el tiempo. hospitalización en la UTI.

Palabras clave: Personal de odontología en hospital, Atención al paciente, Infección hospitalaria.

\section{INTRODUÇÃO}

Nos dias atuais, com novas especializações e enquadramento na equipe interdisciplinar o Cirurgião Dentista (CD) encontra-se em uma nova vivencia clínica, no qual a atividade ocupacional nessa área não possui apenas o cuidado na cavidade oral do paciente, mas sim busca analisar o quadro clínico do paciente como um todo, visando dessa maneira uma interrelação pessoal entre o profissional e o paciente, alcançandose dessa maneira um seguimento relevante para o resultado final satisfatório de ambos. Para que ocorra esse benefício mútuo, o CD tem que estar sempre qualificando-se para obter um domínio em sua área de atuação referente ao ambiente hospitalar nas Unidades de terapia intensiva (VILLAR CC, et al., 2016).

Nessa conjuntura, em ritmo progressivo a Odontologia hospitalar está desenvolvendo-se e recebendo merecido mérito ao trabalhar com a equipe multiprofissional, das quais é necessário, sendo observado sempre uma melhor maneira de lidar com processos terapêuticos com atributos benéficos que visam o bem-estar dos pacientes, principalmente aos que apresentam determinados fatores limitantes, como é o caso da falta de mobilidade para cuidar da saúde bucal e suas devidas importâncias. Diante disso o CD leva um melhor aconchego de maneira completa não só para os pacientes debilitados, mas uma atenção mais harmoniosa com os familiares ou cuidadores que ficam na espera de notícias (LAURENCE B, et al., 2015).

Apesar do exposto, o processo que envolve a higienização da cavidade bucal dos pacientes que se encontram na UTI na maioria das vezes são praticadas pelos profissionais da Enfermagem, que muito das vezes não sabem executar as condutas apropriadas e muitos não seguem os protocolos necessários para a alcance da metodologia padronizada para os devidos cuidados (VILLAR CC, et al., 2016).

Por essa razão, no ano de 2008 houve o estabelecimento de uma Lei oㅡ 2.776/2008, na Câmara dos Deputados, dos quais se referia a essas formas de atuações, onde designa a indispensabilidade da presença no assistencialismo do CD no agrupamento da equipe multiprofissional nas UTI's, propondo um tratamento melhor da integridade da saúde bucal dos pacientes que se encontram hospitalizados. Além de tudo, essa proposta designa que os indivíduos em que situam-se em condições de internamentos sem estarem em uma gravidade maior possuam os mesmos cuidados, objetivando com isso o não agravamento do quadro clínico deles (BRASIL, 2008).

As práticas habituais de atenções na cavidade oral obtém pequena importância e prioridades nas UTIs, consequentemente, existe pouca possibilidade que durante a permanência do paciente nessa condição e meio o processo referente a higienização bucal seja tomada como prioridade, não sendo na maioria das vezes efetivadas da maneira certa, especialmente nos primeiros 3 dias, em que ocorre colonizações por microrganismos gram-negativos das quais estão envoltas por uma cápsula protetora, essa cápsula por sua vez ajuda a evitar que os glóbulos brancos do sangue (que lutam contra infecções) ingiram as bactérias, 
prejudicando ainda mais o quadro clínico do paciente, principalmente aqueles que são intubados e precisam de ventilação de forma mecânica (BAEDER FM, et al., 2012; AMARAL COFD, et al., 2013).

A integralidade da junção multidisciplinar, com profissionais capacitados de distintos âmbitos de atuação presentes no mesmo paciente que demanda atenção de forma elevada, não são encontradas apenas nos locais físicos ou nos equipamentos que irão ser utilizados, o cuidado vai além de todas as tecnologias, é necessário que a equipe esteja completamente capacitada em tomar decisões no momento correto, fundamentando-se em uma precisa percepção e entendimento do estado fisiopatológico do pacientes e dos processos terapêuticos que serão estabelecidos (NAIR GB e NIEDERMAN MS, 2015).

Deste modo, o CD especializado na odontologia hospitalar dispõe como sendo a fundamental desígnio em sua atuação buscar examinar e verificaras apurações dos exames clínicos pertinentes em indivíduos que encontrem-se com enfermidades e estejam hospitalizados objetivando conduzir de forma direta o quadro clínico do paciente, procurando diminuir a probabilidade da alteração da cavidade oral, retirando os prováveis focos de infecções bacterianas que acarretam doenças que ocorrem quando as formas prejudiciais de bactérias se multiplicam no interior da cavidade bucal. Podendo estabelecer infecções irreverssíveis se não forem tratadas de forma rápida e objetiva. Os Cirurgiões Dentistas podem realizar desde uma restauração oral da mais simples até uma cirurgia se necessário for. Desse modo, é necessário que os processos terapêuticos referente a parte medicamentosa e as técnicas abordadas realizados pela equipe médica estejam em concordância com os CD's buscando a melhora do paciente por completo (KIYOSHI-TEO H e BLEGEN M, 2015).

A evolução da saúde bucal realizado por CD's reduz de maneira satisfatória o aparecimento de processos patológicos que são relacionados ao sistema respiratório e a multiplicação de infecções que podem irem para outros órgãos vitais prejudicando assim o bem-estar do paciente, sendo levado em consideração que muito dos agravamentos dos quadros clínicos que são ocorridos não se estabeleceram devidamente por causa da condição clínica introdutória que levou o paciente ao estado em que ele se encontra, muito dos casos que sofrem maior índice de prejuízo pelo descuido da cavidade oral, são pacientes que apresentam maior idade, em particular, os indivíduos que estão no hospital internados por um período grande de tempo nas UTI's (GMUR C, et al., 2013; TAN L, et al., 2014).

Sabendo-se as respectivas importâncias que a Odontologia Hospitalar pode conduzir em forma de vantagens e tendo em vista a elevada relevância do trabalho em grupos interdisciplinares, o respectivo trabalho tem como perspectiva destacar a relevância do Cirurgião Dentista na Unidade de Terapia Intensiva focando-se na execução dos cuidados da cavidade bucal desses pacientes, contando-se da entrada do indivíduo no ambiente hospitalar até os cuidados que são tomados para a sua melhora.

\section{REVISÃO BIBLIOGRÁFICA}

\section{Humanização no atendimento}

No Brasil, as formas de ações que são prestas referente as ocupações referentes ao sistema de saúde estão concernentes pelo meio de um princípio do qual engloba uma rede de atenção básica aos indivíduos. Referente a essa forma de atenção à saúde pode-se observar que nela se constitui por meio de disponibilização de ocupações de serviços dos mais variados na região brasileira, do qual pode-se citar: o atendimento a pacientes que não possuem locomoção sendo feito dessa maneira os primeiros atendimentos no âmbito domiciliar, as unidades de assistências básicas que é a principal porta de entrada com toda a rede de atenção à saúde, Centro especializados em diversas áreas e os hospitais. Essa forma de organização e de exemplo sobretudo estabelecem a padronização das pessoas que possuem um poder aquisitivo menor, dos quais não podem pagar plano de saúde para uma assistência particularizada (COSTA ICC e ARAÚJO MNT, 2011).

É visível a importância em que os hospitais se denotam para a população em um contexto geral, dos quais são representados como micro redes em que beneficiam a sociedade com seu grau de multiprofissionalidade distinta podendo atuar na disponibilização de prestações de serviços de maneira geral, fazendo-se como um 
dos mais relevantes as enfermarias de pronto atendimentos que possuem um grande fluxo de pacientes, as unidades de saúde que possuem sistemas de centros cirúrgicos de maneira especializada, a maternidade e a UTI em que é uma unidade bastante complexa composta de sistemas que fazem o processo de monitorização de maneira contínua em que admite pacientes potencialmente graves ou com descompensação de um ou mais sistemas orgânicos e que com esse suporte e o tratamentos terapêuticos intensivos tenham possibilidade de se recuperar, tendo por tanto uma visão mais cautelosa dos profissionais para o bem-estar e a melhoria do paciente que nela se encontra (BERKEY DB e SCANNAPIECO FA, 2013).

\section{Odontologia hospitalar}

Uma das maneiras que auxiliam de forma direta para o alcance de efeitos suficientes e benéficos dos pacientes que se encontram internados na UTI é a introdução dos Cirurgiões dentistas com especialização em Odontologia Hospitalar. Nos últimos anos vem sendo tramitado diversas diretrizes para a implantação do CD nas UTl's através do Projeto de Lei 2776/2008, dos quais adquiriu frutos positivos através da esfera relacionada a Comissão de Assuntos Sociais com realce na saúde, cujo real significado dessa reunião foi dispor como fundamental intuito a introdução $O C D$ nos hospitais. Levando-se em consideração as competências profissionais que os mesmos estabelecem, esse planejamento efetuado pelo Senado Federal determina regularidade incluindo os Cirurgiões Dentistas da Unidade de Terapia Intensiva, tanto em hospitais públicos quanto os privados tendo como foco o melhoramento dos cuidados na saúde da cavidade oral dos pacientes que se situa em uma UTI (COSTA ICC e ARAÚJO MNT, 2011; LAMBERT ML, et al., 2013).

$\mathrm{Na}$ odontologia existe a especialidade que habilita o Cirurgião Dentista a atuar no âmbito hospitalar reconhecida como odontologia Hospitalar dos quais ela tenciona ao profissional a realizar atenções, consciência e tratamentos na região da cavidade oral dentro dos hospitais, principalmente em pacientes que apresentam pouca ou nenhuma mobilidade (COSTA ICC e ARAÚJO MNT, 2011; LAMBERT ML, et al., 2013). O início das prestações de serviços dos CD's em ambientes hospitalares iniciou-se em 1901, em Filadélfia, em que dispuseram como funcionalidade medidas de estabelecimentos as atenções dentais dos pacientes que lá estavam além de dispor de capacitar os discentes da campo de atuação (MIRANDA AF, et al., 2016).

Em trabalhos científicos a especialização da Odontologia Hospitalar é salientada como uma existência que cuida de procedimentos que abrangem desde pequenas complexidades até a altas, dos quais busca de forma ética e continua o tratamento dos pacientes com procedimentos terapêuticos, tendo como foco a melhora dos pacientes, evitando assim estipuladas patologias que venham a existir, como é o caso de problemas periodontais e respiratórios prejudicando com isso ainda mais o quadro clínico do paciente. Recomenda-se que a forma de comunicação com o paciente tem que ser estabelecida com um olhar geral do quadro clínico, sendo visto o mesmo como um todo, e não apenas analisando o problema especifico que o levou a ficar internado no hospital, os profissionais da área de saúde vem ter uma visão não apenas especifica de cada área, buscando desta forma uma interação multiprofissional, essa forma de manejo faz com quer os profissionais tenham resultados mais benéficos e de menor tempo (MARTA SN, 2011; JANSSON MM, et al., 2017).

Devido ser reconhecido no Brasil como uma área nova de atuação o Cirurgião Dentista deve portar-se de um conhecimento bastante aprofundado sobre a vivencia no ambiente hospitalar, visto que muitos profissionais conhecem muito bem como funciona o desempenho em tratamentos realizados em clínicas odontológicas, consultórios particulares e até mesmo em Unidades Básicas de Saúde, por isso é necessário a especialização porque a atenção, ao paciente é bem maior e requer um cuidado diferenciado, a grau de experiências vivenciadas em discentes a concepção e perspectiva em meios hospitalares continua sendo insatisfatório e quando possui, a quantidade de cargas horarias necessárias são insuficientes para observar e entender toda logística de como funciona um ambiente hospitalar e o verdadeiro papel que o Cirurgião dentista tem que executar (JANSSON MM, et al., 2017).

\section{Ambiente hospitalar}

O Cirurgião dentista realiza suas experiências nos hospitais como sendo um orientador e solicitador de prestações de serviços que envolvem a integridade da cavidade oral como toda, assim como analisa os 
exames laboratoriais dos pacientes que os mesmos acompanham seja ele a nível apenas da permanência de pacientes por pouco tempo ou tempo indeterminado, esse trabalho é realizado frequentemente, visando desta maneira assegurar, disponibilizar a integridade da melhora dos pacientes, ao qual é classificado como uma inovação de bastante valor na nova realidade hospitalar (BLUM DF, et al., 2017).

\section{Os impactos dos cirurgiões dentistas no ambiente hospitalar}

Nos últimos anos pesquisas foram realizadas em diversos Hospitais renomados que possuíam um alto índice de internações em UTl's nas análises feitas das pesquisas um dos principais objetivos era observar e examinar a situação de saúde da cavidade oral dos pacientes, procurando explorar a interrelação bilateral entre as situações bucais e os desenvolvimentos dos tratamentos terapêuticos realizados pela equipe medica, contemplando desta maneira os resultados que demonstraram que com o elevado nível de estresse que o paciente passa nos hospitais, especialmente aqueles indivíduos que apresentam idades avançadas e tomam medicamentos contínuos e não possuem os cuidados na limpeza da cavidade oral, apresentam graves problemas relacionados a bactérias no sistema da cavidade oral, mudando assim as propriedades normais que deveria ser estabelecidas, além do problema no potencial hidroxiliônico $(\mathrm{pH})$, xerostomia, tendo como consequência o elevado índice de infecções e piora no quadro clínico do paciente, ocorrendo uma elevada preocupação nos resultados que foram estabelecidos nessa pesquisa. Assim sendo, chegaram a uma conclusão demonstrando como é de suma importância o assistencialismo do CD nos hospitais, principalmente nas UTI's (LIMA DC, et al., 2011; RODRIGUES SS, et al., 2016).

Conforme o decreto estabelecido pelo Código de Ética da Odontologia, é de competência dos profissionais cirurgião dentista que possuem especialização em Odontologia Hospitalar, inserir o paciente a internação, além de fazer o acompanhamento dos pacientes não só em consultórios ou Unidade Básicas de Saúde como também em hospitais de que apresentam grande porte seja ele público ou privado, com ou sem fins lucrativos (associação ou fundação), criadas com o objetivo de produzir o bem, concernindo as diretrizes técnicas administrativas das instituições que esses profissionais prestam serviços (CFO, 2016).

\section{Relação entre doenças bucais e sistêmicas}

O início dos questionamentos ao que se refere as interrelações das patologias orais e doenças sistêmicas aconteceram desde o princípio do domínio do entendimento da medicina na sociedade (ORY J, et al., 2018). Desde esses momentos, inúmeros pesquisadores da comunidade cientifica propuseram-se em alcançar desfechos que evidenciem a relação existente entre essas ocorrências, designando que questões relacionadas a cavidade oral como é o caso da periodontite que pode desencadear até mesmo a perda do elemento dentário se não for tratada, além de demonstrar com clareza e objetividade que ela é um dos grandes fatores de risco principalmente ao que se refere a doenças cardíacas e pulmonares. Isso acontece porque seu foco possui atividades de espalhamento dos agentes patogênicos na corrente sanguínea ocasionando problemas sérios de forma sistêmica (ALOTAIBI AK, et al., 2014).

O sistema que envolve a cavidade oral retrata uma das maiores formas de comunicabilidade do meio externo do corpo humano com a parte interna, dispondo em média de 500 distintas variedades de microrganismos dos quais possuem potencial de realizarem o processo de colonização na área em que eles se encontrarem. Ocasionando problemas nos mecanismos normais de defesas contra infecção debilitando ainda mais os pacientes (LAURENCE B, et al., 2015; ORY J, et al., 2018). Dos enfermos presentes na UTI, várias dessas microbiotas presentem expõem sua potencialidade de patogenia expandida, portando a problemas periodontais de maneira severas podendo chegar a um quadro de candidíase.

Entre as enfermidades encontradas de maneira sistêmica que predispõe a condições de riscos para a contração da patologia periodontal estão os paciente que apresentam diabetes, sendo observado que nessas pessoas a forma de manifestações ocorrem de maneira mais grave por causa de relações estabelecidas por conta do metabolismo e a AIDS que, por causa do processo de Imunodeficiências Primárias, conduz a ocorrência de processos infecciosos de vários fungos, bactérias e até mesmo outros vírus para o paciente que está sendo atendido pela equipe multiprofissional (BLUM DF, et al., 2017). A base fisiológica e sistêmica do corpo humano é estabelecida por inúmeros plexos de sistemas e órgãos em que a cavidade oral dispõe 
de maior número de dimensão de distintas diversidade taxonômica de gêneros/espécies de micróbios dos quais Incluem os vírus, as bactérias, os protozoários, fungos e os ácaros, sendo que existem algumas espécies que são prejudiciais ao ser humano. Quando a microbiota da boca está apresentando uma condição favorável, melhor dizendo, costumeiro, dos quais disponibilizam um bom e natural equilíbrio, regularidade, proporcionalidade com o hospedeiro, contribuindo com seu estado de integralidade, garantido mediante políticas sociais e econômicas que evitem o risco do agravo à saúde tanto de forma física quanto de maneira psicológica (VILLAR CC, et al., 2016).

Devem serem levadas em consideração as seriedades das particularidades anatômicas e fisiológicas da cavidade bucal de cada paciente visto que elas apresentam formas particularizadas sendo distintas de um indivíduo para o outro, destacando desta forma sua amplitude, em razão que a cavidade bucal dispõe de inúmeras diversidades de formas estruturais e tecidos que têm potencial de sofrerem modificações por causa dos níveis de oxigenação presentes no local, proporções de nutrientes disponíveis, elementos deliberativos como a etimologia e condições imunológicas que se fundamenta na ativação das células efetoras dos hospedeiros. A cavidade oral, mais precisamente a língua é uma área úmida em que retém muito sistema onde os microrganismos se multiplicam-se com elevada facilidade e vigor especialmente na área das papilas linguais e do dorso, do qual inúmeros microrganismos agressores vão em direção para os revestimentos das camadas gengivais (BLUM DF, et al., 2017).

\section{Cuidados com os pacientes}

Desta maneira consegue-se perceber que os enfermos em que situam-se com um nível de mal-estar bastante agravado, especialmente os pacientes que encontram-se nas UTI's necessitam de uma forma diferenciada ao que se refere ao processo de cautela, dos quais os Cirurgiões dentistas precisam intensificar as observações de maneira cuidadosa, visto que à imensa dimensão de agentes infecciosos presentes no local da cavidade bucal dispõem de uma ampla multiplicação de forma acelerada, e uma das causas que ocorrem esse vasto processo de ocupação decorre devido à fatores estabelecidos pelo sistema respiratório (BLUM DF, et al., 2017).

É bastante relevante considerar todos os protocolos que levam a limpeza da cavidade bucal para se estabelecer técnicas corretivas para eliminar definitivamente qualquer possibilidade de criação de qualquer microrganismo em que prejudique os pacientes, para isso é necessário ser feito profilaxias na cavidade bucal, ter controle em pacientes que apresentam corrimentos salivares com abundancia, analises corriqueiras de presença de biofilmes, analisar a limpeza da língua, principalmente a parte onde encontra-se as glândulas salivares e as papilas localizadas no dorso da língua, se necessário for é importante fazer uma limpeza com utilização de clorexidina a $0,12 \%$, tendo em vista que sua utilização deve ser realizada com cuidado e sobre a supervisão do Cirurgião Dentista. Além da conta, quando o paciente apresenta disposição ajudar ao mesmo a executar o processo de escovação dentaria e explicar a ele toda a importância que esse ato possui (MIRANDA AF, et al., 2016; VILLAR CC, et al., 2016).

\section{Infecções sistêmicas causadas por microrganismos bucais}

Em análises feitas em pacientes que estavam internados em Unidades de Terapia Intensiva foram observados inúmeros microrganismos, dos quais pode-se comprovar que a microbiota presentes na cavidade oral possuem potencial de promover contratempos de maneira alarmante ao que se refere a infecções em órgãos distanciados, dos quais pode ser analisado as infecções formadas por microrganismos causadores (GMUR C, et al., 2013) (Quadro 1). 
Quadro 1 - Infecções sistêmicas estabelecidas através da microbiota da cavidade oral.

\begin{tabular}{|c|c|}
\hline Infecções & Microrganismos \\
\hline $\begin{array}{c}\text { Endocardite infecciosa (ocorre quando uma bactéria entra na } \\
\text { corrente sanguínea e vai para as válvulas previamente lesionadas, } \\
\text { aderindo-se a elas). }\end{array}$ & $\begin{array}{c}\text { Estreptococos bucais E. corrodens } \\
\text { A. actinomycetemcomitans M. } \\
\text { micros }\end{array}$ \\
\hline $\begin{array}{c}\text { Bacteremia (Geralmente não causa sintomas, mas, às vezes, } \\
\text { acumulam-se bactérias em certos tecidos ou órgãos, causando } \\
\text { infecções sérias). }\end{array}$ & $\begin{array}{c}\text { Estreptococos bucais P. gingivalis } \\
\text { Enterobacteriaceae Candida sp. }\end{array}$ \\
\hline $\begin{array}{c}\text { Sepse (A sepse é um conjunto de manifestações graves em todo o } \\
\text { organismo produzidas por uma infecção). }\end{array}$ & A. actinomycetemcomitans \\
\hline $\begin{array}{c}\text { Abscesso Cerebral (Pus intracerebral por causa da intercorrência } \\
\text { de grande coleção de bactérias). }\end{array}$ & A. actinomycetemcomitans \\
\hline $\begin{array}{c}\text { Infecções respiratórias (Infecção na região respiratória, que atinge } \\
\text { desde as vias aéreas superiores quanto as vias aéreas inferiores). }\end{array}$ & $\begin{array}{c}\text { A. actinomycetemcomitans } \\
\text { Enterobacteriaceae } \\
\text { Staphylococcus }\end{array}$ \\
\hline $\begin{array}{c}\text { Infecções intra-abdominais (Doença inflamatória pélvica É uma } \\
\text { síndrome clínica causada por vários microrganismos, que ocorre } \\
\text { devido à entrada de agentes infecciosos). }\end{array}$ & P. gingivalis \\
\hline $\begin{array}{c}\text { Oftalmoplegia (O comprometimento do movimento na região } \\
\text { horizontal dos olhos). }\end{array}$ & A. actinomycetemcomitans \\
\hline Conjuntivite crônica (infecção na região ocular). & M. micros \\
\hline $\begin{array}{c}\text { Endoftalmite (Ocorrida no interior dos olhos, através de infecção na } \\
\text { corrente sanguínea). }\end{array}$ & A. actinomycetemcomitans \\
\hline $\begin{array}{c}\text { Abscesso do tubo ovariano (Secreções que ocasionam problemas } \\
\text { purulentos). }\end{array}$ & P. gingivalis \\
\hline
\end{tabular}

Fonte: NETO JMAS et al, 2020. Modificada de GMUR C, et al., 2013; ALOTAIBI AK, et al., 2014; VILLAR CC, et al., 2016.

Nas atuações estabelecidas pela Política Nacional de Saúde Bucal (PNSB) as equipes de saúde devem trabalharem de forma multiprofissional respeitando uma norma em que expõe a importância dessa relação profissional, buscando um melhor desenvolvimento técnico e estabelecendo melhores desempenhos referentes aos trabalhos oferecidos a população, com isso é de suma relevância a presença do CD para os cuidados da saúde bucal de todos os pacientes, independente do estado de saúde que ele encontre-se no hospital. Nessa continuidade, os trabalhos relacionados aos serviços de saúde carecem do acompanhamento de forma conjunta, sempre analisando não só o motivo que levou o paciente até ao hospital, mas possuir um cuidado como um todo, iniciando pela boca do paciente, evitando assim a proliferação de microrganismos indesejáveis, prejudicando ainda mais a condição de integridade do paciente (CORACIN FL, 2012).

\section{CONSIDERAÇÕES FINAIS}

Nos dias atuais o CD vem buscando se qualificar ainda mais nas intervenções dos processos reabilitadores em indivíduos que encontram-se na UTI, para isso esses profissionais qualificam-se com a especialização em Odontologia Hospitalar, com essa qualificação procuram diminuir focos infecciosos na cavidade bucal, realizando diversos procedimentos, melhorando dessa forma o quadro clinico de saúde do paciente e diminuindo o tempo de internação dos mesmos nas Unidades de terapia Intensiva, objetivando dessa forma diminuir focos de microrganismos que possam a vim causar prejuízo na saúde dos pacientes através de infecções da cavidade oral, realizando exames e estabelecendo condutas como cuidados periodontais, restabelecimento da integridade dos elementos dentais, cuidados na microbiota, no $\mathrm{pH}$ do meio bucal e procedimentos cirúrgicos com a compreensão de minimizar o tempo de internação desses pacientes. $O$ Cirurgião Dentista cada vez mais vem ganhando espaço nos ambientes hospitalares, visto que os pacientes são beneficiados e os índices de infecções principalmente as respiratórias são reduzidas, como pode ser analisado nas revisões de literaturas citadas no artigo. 


\section{REFERÊNCIAS}

1. ALOTAIBI AK, et al. Does the presence of oral care guidelines affect oral care delivery by intensive care unit nurses? A survey of Saudi intensive care unit nurses. Am J Infect Control. 2014;42(8):921-2

2. AMARAL COFD, et al. Importância do cirurgião-dentista em Unidade de Terapia Intensiva: avaliação multidisciplinar. Rev Assoc Paul Cirurg Dent, 2013; 67(2):107-111.

3. BAEDER FM, et al. Condição odontológica em pacientes internados em unidade de terapia intensiva. Pesq Bras Odontop Clin Integr, 2012; 12(4): 517-520.

4. BERKEY DB, SCANNAPIECO FA. Medical considerations relating to the oral health of older adults. Spec Care Dentist, Malden, 2013; v. 33, n. 4; 164-176.

5. BLUM DF, et al. Influence of dentistry professionals and oral health assistance protocols on intensive care unit nursing staff. A survey study. Rev Bras Ter Intensiva. 2017;29(3):391-3.

6. BRASIL. Ministério da Saúde. Agência Nacional de Vigilância Sanitária. Resolução-RDC No7, de 24 de Fevereiro de 2010. Dispõe sobre os requisitos mínimos para o funcionamento de Unidades de Terapia Intensiva e dá outras providências. Diário Oficial da União. DOU de 25 de fevereiro de 2010.

7. CFO - CONSELHO FEDERAL DE ODONTOLOGIA. Código de Ética Odontológico. Brasília; 2012. Cap. XI, Artigo 26.

8. CORACIN FL. Interdisciplinaridade. Medicina Bucal: a prática da Odontologia Hospitalar. São Paulo: Santos, 2012. p.25-27, 315p.

9. COSTA ICC, ARAÚJO MNT. Definição do perfil de competências em saúde coletiva a partir da experiência de cirurgiões dentistas atuantes no serviço público. Cien Saude Colet, Rio de Janeiro, v.16, supl.1, 2011, p.1181-1189.

10. GMUR C, et al. Survey on oral hygiene measures for intubated patients in Swiss intensive care units. Schweiz Monatsschr Zahnmed. 2013;123(5):394-409.

11. JANSSON MM, et al. Effects of simulation education on oral care practices - a randomized controlled trial. Nurs Crit Care. 2017;22(3):161-8.

12. KIYOSHI-TEO H, BLEGEN M. Influence of Institutional Guidelines on Oral Hygiene Practices in Intensive Care Units. Am J Crit Care. 2015;24(4):309-18.

13. LAMBERT ML, et al. Prevention of ventilator-associated pneumonia in intensive care units: an international online survey. Antimicrob Resist Infect Control. 2013;2(1):9

14. LAURENCE B, et al. Hospital admissions for pneumonia more likely with concomitant dental infections. Clin Oral Investig. 2015; 19(6):1261-8.

15. LIMA DC, et al. A importância da saúde bucal na ótica de pacientes hospitalizados. Ciênc. Saúde Coletiva. 2011; V.16, suppl. 1;1173-1180.

16. MARTA SN. Programa de assistência odontológica ao paciente especial: uma experiência de 13 anos. RGO- Rev Gaúcha Odontol., Porto Alegre, v.59, n.3, 2011, p.379-385.

17. MIRANDA AF, et al. Oral care practices for patients in Intensive Care Units: A pilot survey. Indian J Crit Care Med, 2016; 20(5): 267-273.

18. NAIR GB, NIEDERMAN MS. Ventilator-associated pneumonia: present understanding and ongoing debates. Intensive Care Med. 2015;41(1):34-48.

19. ORY J, et al. Cost assessment of a new oral care program in the intensive care unit to prevent ventilator-associated pneumonia. Clin Oral Investig. 2018;22(5):1945-51.

20. RODRIGUES SS, et al. Knowledge, Attitude and Practice of the Nursing Team Regarding Oral Health Care in Intensive Care Units in a Reference Hospital of Recife, Brazil. Braz Res Ped Dent Int Clin, 2016; 16(1): 129-139.

21. TAN L, et al. rDNA-based metagenomic analysis of dental plaque and lung bacteria in patients with severe acute exacerbations of chronic obstructive pulmonary disease. J Periodontal Res. 2014;49(6):760-9.

22. VILLAR CC, et al. Effectiveness of intraoral chlorhexidine protocols in the prevention of ventilator-associated pneumonia: meta-analysis and systematic review. Respir Care. 2016;61(9):1245-59. 